\title{
Tetravalent 2-arc-transitive Cayley graphs on non-abelian simple groups
}

\author{
Jia-Li Du, Yan-Quan Feng* \\ Department of Mathematics, Beijing Jiaotong University, Beijing 100044, China
}

\begin{abstract}
A graph $\Gamma$ is said to be 2-arc-transitive if its full automorphism group $\operatorname{Aut}(\Gamma)$ has a single orbit on ordered paths of length 2 , and for $G \leq \operatorname{Aut}(\Gamma), \Gamma$ is $G$-regular if $G$ is regular on the vertex set of $\Gamma$. Let $G$ be a finite non-abelian simple group and let $\Gamma$ be a connected tetravalent 2-arc-transitive $G$-regular graph. In 2004, Fang, Li and $\mathrm{Xu}$ proved that either $G \unlhd \operatorname{Aut}(\Gamma)$ or $G$ is one of 22 possible candidates. In this paper, the number of candidates is reduced to 7 , and for each candidate $G$, it is shown that $\operatorname{Aut}(\Gamma)$ has a normal arc-transitive non-abelian simple subgroup $T$ such that $G \leq T$ and the pair $(G, T)$ is explicitly given.
\end{abstract}

Keywords: Cayley graph, coset graph, simple group.

2010 Mathematics Subject Classification: 05C25, $20 \mathrm{~B} 25$.

\section{Introduction}

Throughout this paper, all groups and graphs are finite, and all graphs are simple and undirected. Let $G$ be a permutation group on a set $\Omega$ and let $\alpha \in \Omega$. Denote by $G_{\alpha}$ the stabilizer of $\alpha$ in $G$, that is, the subgroup of $G$ fixing the point $\alpha$. The group $G$ is semiregular if $G_{\alpha}=1$ for every $\alpha \in \Omega$, and regular if $G$ is transitive and semiregular.

For a graph $\Gamma$, denote by $V(\Gamma), E(\Gamma)$ and $\operatorname{Aut}(\Gamma)$ its vertex set, edge set and full automorphism group, respectively. An $s$-arc in $\Gamma$ is an ordered $(s+1)$-tuple $\left(v_{0}, v_{1}, \ldots, v_{s}\right)$ of vertices of $\Gamma$ such that $v_{i-1}$ is adjacent to $v_{i}$ for $1 \leq i \leq s$, and $v_{i-1} \neq v_{i+1}$ for $1 \leq i<s$. Let $G \leq \operatorname{Aut}(\Gamma)$. The graph $\Gamma$ is said to be $(G, s)$-arc-transitive or $G$-regular if $G$ has a single orbit on $s$-arcs of $\Gamma$ or is regular on vertices of $\Gamma$, respectively. For short, a 1arc means an arc, and $(G, 1)$-arc-transitive means $G$-arc-transitive. The graph $\Gamma$ is said to be $s$-arc-transitive if it is $(\operatorname{Aut}(\Gamma), s)$-arc-transitive. In particular, 0 -arc-transitive is vertex-transitive, and 1-arc-transitive is arc-transitive or symmetric.

For a group $G$ and a subset $\mathrm{S}$ of $G$ such that $1 \notin S$ and $S^{-1}=S$, the Cayley graph $\operatorname{Cay}(G, S)$ on $G$ with respect to $S$ is defined to have vertex set $G$ and edge set $\{\{g, s g\} \mid g \in G, s \in S\}$. For $g \in G$, the map $R(g): x \mapsto x g$ for $x \in G$ is a permutation

${ }^{*}$ Corresponding author. E-mails: JiaLiDu@bjtu.edu.cn, yqfeng@bjtu.edu.cn 
on $G$, and $R(G)=\{R(g) \mid g \in G\}$ consists of a permutation group on $G$, called the right regular representation of $G$. It is easy to see that $R(G) \leq \operatorname{Aut}(\Gamma)$. A Cayley graph $\Gamma=\operatorname{Cay}(G, S)$ is said to be normal if $R(G)$ is a normal subgroup of Aut $(\Gamma)$, and in this case, $\operatorname{Aut}(\Gamma)=R(G) \rtimes \operatorname{Aut}(G, S)$ by Godsil [6] or Xu [20], where $\operatorname{Aut}(G, S)=\{\alpha \in$ $\left.\operatorname{Aut}(G) \mid S^{\alpha}=S\right\}$. Note that $\operatorname{Cay}(G, S)$ is $R(G)$-regular. It is well-known that if $\Gamma$ is $G$-regular then $\Gamma$ is isomorphic to a Cayley graph on $G$.

The investigation of symmetric graphs has a long and interesting history, highlighted at an early stage by ingenious work by Tutte [23, 24] on the cubic case. Let $\Gamma$ be a connected symmetric cubic Cayley graph on a non-abelian simple group G. Li [10] proved that either $\Gamma$ is normal or $G=A_{5}, A_{7}, \operatorname{PSL}(2,11), M_{11}, A_{11}, A_{15}, M_{23}, A_{23}$ or $A_{47}$. Based on Li's work, $\mathrm{Xu}$ et al [21, 22] proved that either $\Gamma$ is normal or $G=A_{47}$, and for the latter, $\Gamma$ is 5 -arc-transitive and there are only two such graphs.

Let $\Gamma$ be a connected tetravalent 2-arc-transitive Cayley graph on a non-abelian simple group $G$. Fang et al [3] proved that either $\Gamma$ is normal, or $G$ is one of the 22 possible candidates listed in [3, Table 1] (the group $A_{23}$ is missed in the table). The following is the main result of this paper.

Theorem 1.1 Let $G$ be a non-abelian simple group and $\Gamma$ a connected tetravalent 2-arctransitive $G$-regular graph. Then either $G \unlhd \operatorname{Aut}(\Gamma)$ or $\operatorname{Aut}(\Gamma)$ contains a normal arctransitive non-abelian simple subgroup $T$ such that $G \leq T$ and $(G, T)$ is listed in Table 1 .

\begin{tabular}{|c|c|c|c|c|c|c|c|}
\hline$G$ & $M_{11}$ & $A_{2^{3} \cdot 3-1}$ & $A_{2^{2} \cdot 3^{2}-1}$ & $A_{2^{3} \cdot 3^{2}-1}$ & $A_{2^{4} \cdot 3^{2}-1}$ & $A_{2^{4} \cdot 3^{3}-1}$ & $A_{2^{4} \cdot 3^{6}-1}$ \\
\hline$T$ & $M_{12}$ & $A_{2^{3} \cdot 3}$ & $A_{2^{2} \cdot 3^{2}}$ & $A_{2^{3} \cdot 3^{2}}$ & $A_{2^{4} \cdot 3^{2}}$ & $A_{2^{4} \cdot 3^{3}}$ & $A_{2^{4} \cdot 3^{6}}$ \\
\hline
\end{tabular}

Table 1: 7 possible pairs of non-abelian simple groups

For connected symmetric cubic Cayley graphs on non-abelian simple groups, similar to Theorem 1.1, there are six possible pairs $(G, T)=\left(A_{47}, A_{48}\right),\left(\operatorname{PSL}(2,11), M_{11}\right)$, $\left(M_{11}, M_{12}\right),\left(A_{11}, A_{12}\right),\left(M_{23}, M_{24}\right)$ or $\left(A_{23}, A_{24}\right)$ (see [10, Theorem 7.1.3]), and Xu et al [21, 22] proved that only the pair $(G, T)=\left(A_{47}, A_{48}\right)$ can happen and there are exactly two connected non-normal symmetric cubic Cayley graphs on $A_{47}$. For the 7 possible pairs of $(G, T)$ in Theorem 1.1, by MAGMA [1] there is only one 2-arc-transitive Cayley graph on $M_{11}$ for $(G, T)=\left(M_{11}, M_{12}\right)$ (see Remark of Lemma [3.2), and there are four 2-arctransitive Cayley graphs on $A_{23}$ for $(G, T)=\left(A_{23}, A_{24}\right)$. The number of 3 -arc-transitive Cayley graphs on $A_{35}$ for $(G, T)=\left(A_{35}, A_{36}\right)$ is 4 , on $A_{71}$ for $(G, T)=\left(A_{71}, A_{72}\right)$ is 18 , and on $A_{143}$ for $(G, T)=\left(A_{143}, A_{144}\right)$ is 31 . At the end of this paper, we give examples of connected non-normal 2-arc-transitive Cayley graph on $A_{23}$ for $(G, T)=\left(A_{23}, A_{24}\right)$ (see Example 3.4).

\section{Preliminaries}

In this section, we describe some preliminary results which will be used later. The first result is the stabilizers of tetravalent 2-arc-transitive graphs, given in [14, Theorem 4]. 
Proposition 2.1 Let $\Gamma$ be a connected tetravalent $(G, s)$-arc-transitive but not $(G, s+1)$ arc-transitive graph with $v \in V(\Gamma)$. If $s \geq 2$ then one of the following occurs:

(1) For $s=2$, we have $G_{v} \cong A_{4}$ or $S_{4}$. In particular, $\left|G_{v}\right|=2^{2} \cdot 3$ or $2^{3} \cdot 3$.

(2) For $s=3$, we have $G_{v} \cong \mathbb{Z}_{3} \times A_{4}, \mathbb{Z}_{3} \rtimes S_{4}$, or $S_{3} \times S_{4}$. In particular, $\left|G_{v}\right|=2^{2} \cdot 3^{2}$, $2^{3} \cdot 3^{2}$ or $2^{4} \cdot 3^{2}$.

(3) For $s=4$, we have $G_{v} \cong \mathbb{Z}_{2}^{3} \rtimes \operatorname{GL}(2,3)=\operatorname{AGL}(2,3)$. In particular, $\left|G_{v}\right|=2^{4} \cdot 3^{3}$.

(4) For $s=7$, we have $G_{v} \cong \mathbb{Z}_{3}^{5} \rtimes \mathrm{GL}(2,3)$. In particular, $\left|G_{v}\right|=2^{4} \cdot 3^{6}$.

Remark: For $s=7$, by [14, Theorem 1.1], $G_{v}=\left\langle e_{0}, e_{1}, e_{2}, e_{3}, e_{4}, e_{5}, e_{6}, d\right\rangle \cong \mathbb{Z}_{3}^{5} \rtimes \mathrm{GL}(2,3)$ with the following relations: $e_{i}^{3}=d^{2}=1$ for all $0 \leq i \leq 6,\left[e_{i}, e_{j}\right]=1$ for all $|i-j|<4$, $\left[e_{0}, e_{4}\right]=e_{2},\left[e_{0}, e_{5}\right]=e_{1}^{-1} e_{2} e_{3}^{-1} e_{4}^{-1}, e_{0} e_{6} e_{0}=e_{6} e_{0} e_{6},\left[e_{1}, e_{5}\right]=e_{3}, e_{1}^{e_{6}}=e_{5} e_{4} e_{3}^{-1} e_{2} e_{1}$, $e_{2}^{e_{6}}=e_{2} e_{4}^{-1}, e_{k}^{d}=e_{k}^{-1}$ for $k=0,2,3,5,6$ and $e_{l}^{d}=e_{l}$ for $l=1,4$, and by MAGMA [1], $G_{v}$ has no normal subgroup isomorphic to $A_{4}, S_{4}, \mathbb{Z}_{3} \times A_{4}, \mathbb{Z}_{3} \rtimes S_{4}, S_{3} \times S_{4}$, AGL(2,3), or a non-trivial 2-group.

Let $\Gamma$ be a graph and let $N \leq \operatorname{Aut}(\Gamma)$. The quotient graph $\Gamma_{N}$ of $\Gamma$ relative to $N$ is defined as the graph with vertices the orbits of $N$ on $V(\Gamma)$ and with two orbits adjacent if there is an edge in $\Gamma$ between those two orbits. The theory of quotient graph is widely used to investigate symmetric graphs. The following proposition can be deduced from [5, Theorem 1.1] and [15, Theorem 4.1].

Proposition 2.2 Let $\Gamma$ be a connected tetravalent $(G, s)$-arc-transitive graph for some $s \geq 2$ and let $N$ be a normal subgroup of $G$. If $N$ is transitive then either $N$ is regular or arc-transitive, and if $N$ has at least three orbits then $N$ acts semiregularly on $V(\Gamma)$ and the quotient graph $\Gamma_{N}$ is a connected tetravalent $(G / N, s)$-arc-transitive graph.

Guralnick [7] classified non-abelian simple groups which contain subgroups of index a power of a prime, and by [11, Theorem 1], we have the following result.

Proposition 2.3 Let $G$ and $T$ be non-abelian simple groups such that $G \leq T$ and $\mid T$ : $G \mid=2^{a} \cdot 3^{b} \geq 6$ with $0 \leq a \leq 4$ and $0 \leq b \leq 6$. Then $T, G$ and $|T: G|$ are listed in Table Q

\begin{tabular}{|c|c|c||c|c|c|}
\hline$T$ & $G$ & $|T: G|$ & $T$ & $G$ & $|T: G|$ \\
\hline$M_{11}$ & $\operatorname{PSL}(2,11)$ & $2^{2} \cdot 3$ & $M_{12}$ & $M_{11}$ & $2^{2} \cdot 3$ \\
\hline$M_{24}$ & $M_{23}$ & $2^{3} \cdot 3$ & $\operatorname{PSU}(3,3)$ & $\operatorname{PSL}(2,7)$ & $2^{2} \cdot 3^{2}$ \\
\hline$A_{9}$ & $A_{7}$ & $2^{3} \cdot 3^{2}$ & $\operatorname{PSp}(4,3)$ & $A_{6}$ & $2^{3} \cdot 3^{2}$ \\
\hline $\operatorname{PSp}(6,2)$ & $A_{8}$ & $2^{3} \cdot 3^{2}$ & $\operatorname{PSU}(4,3)$ & $\operatorname{PSL}(3,4)$ & $2 \cdot 3^{4}$ \\
\hline$M_{12}$ & $\operatorname{PSL}(2,11)$ & $2^{4} \cdot 3^{2}$ & $\operatorname{PSU}(4,3)$ & $A_{7}$ & $2^{4} \cdot 3^{4}$ \\
\hline$G_{2}(3)$ & $\operatorname{PSL}(2,13)$ & $2^{4} \cdot 3^{5}$ & $A_{n}$ & $A_{n-1}$ & $n=2^{a} \cdot 3^{b}$ \\
\hline
\end{tabular}

Table 2: Non-abelian simple group pairs of index $2^{a} \cdot 3^{b}$ 
Let $G$ and $E$ be two groups. We call an extension $E$ of $G$ by $N$ a central extension of $G$ if $E$ has a central subgroup $N$ such that $E / N \cong G$, and if further $E$ is perfect, that is, the derived group $E^{\prime}=E$, we call $E$ a covering group of $G$. A covering group $E$ of $G$ is called a double cover if $|E|=2|G|$. Schur [17] proved that for every non-abelian simple group $G$ there is a unique maximal covering group $M$ such that every covering group of $G$ is a factor group of $M$ (see [8, Kapitel V, §23]). This group $M$ is called the full covering group of $G$, and the center of $M$ is the Schur multiplier of $G$, denoted by $\operatorname{Mult}(G)$.

Proposition 2.4 For $n \geq 5$, the alternating group $A_{n}$ has a unique double cover $2 . A_{n}$, and for $n \geq 7$, all subgroups of index $n$ of $2 . A_{n}$ are conjugate and isomorphic to $2 . A_{n-1}$.

Proof: By Kleidman and Liebeck [9, Theorem 5.1.4], Mult $\left(A_{n}\right) \cong \mathbb{Z}_{2}$ for $n \geq 5$ with $n \neq 6,7$, and $\operatorname{Mult}\left(A_{n}\right) \cong \mathbb{Z}_{6}$ for $n=6$ or 7 . This implies that $A_{n}$ has a unique double cover for $n \geq 5$, and we denote it by $2 . A_{n}$. Since $A_{n}$ has no proper subgroup of index less than $n$, all subgroups of index $n$ of $2 . A_{n}$ contain the center of order 2 of $2 . A_{n}$. Let $n \geq 7$. By [19, 2.7.2], 2. $A_{n}$ contains a subgroup 2. $A_{n-1}$ of index $n$, and since all subgroups of index $n$ of $A_{n}$ are conjugate, all subgroups of index $n$ of $2 . A_{n}$ are conjugate and hence isomorphic to $2 . A_{n-1}$.

Now, we introduce the so called coset graph. Let $G$ be a group and $H \leq G$. Denote by $D$ a union of some double cosets of $H$ in $G$ such that $D^{-1}=D$. The coset graph $\Gamma=$ $\operatorname{Cos}(G, H, D)$ on $G$ with respect to $H$ and $D$ is defined to have vertex set $V(\Gamma)=[G: H]$, the set of right cosets of $H$ in $G$, and the edge set $E(\Gamma)=\{\{H g, H d g\} \mid g \in G, d \in D\}$. It is well known that $\Gamma=\operatorname{Cos}(G, H, D)$ has valency $|D| /|H|$ and it is connected if and only if $G=\langle D, H\rangle$, that is, $D$ and $H$ generate $G$. The action of $G$ on $[G: H]$ by right multiplication induces a vertex-transitive group of automorphisms of $\Gamma$, and this group is arc-transitive if and only if $D$ is a single double coset. Moreover, this action is faithful if and only if $H_{G}=1$, where $H_{G}$ is the largest normal subgroup of $G$ contained in $H$. Clearly, $\operatorname{Cos}(G, H, D) \cong \operatorname{Cos}\left(G, H^{\alpha}, D^{\alpha}\right)$ for each $\alpha \in \operatorname{Aut}(G)$.

Conversely, let $\Gamma$ be a $G$-vertex-transitive graph. By [16], $\Gamma$ is isomorphic to a coset graph $\operatorname{Cos}(G, H, D)$, where $H=G_{v}$ is the vertex stabilizer of $v \in V(\Gamma)$ in $G$ and $D$ consists of all elements of $G$ which map $v$ to one of its neighbors. It is easy to show that $H_{G}=1$ and $D$ is a union of some double cosets of $H$ in $G$ satisfying $D^{-1}=D$. Assume that $G$ is arc-transitive and $g \in G$ interchanges $v$ and one of its neighbors. Then $g^{2} \in H$ and $D=H g H$. Furthermore, $g$ can be chosen as a 2-element in $G$, and the valency of $\Gamma$ is $|D| /|H|=\left|H: H \cap H^{g}\right|$. For more details regarding coset graph, refer to [4, 12, 13, 16].

Proposition 2.5 Let $\Gamma$ be a connected $G$-arc-transitive graph and $\{u, v\}$ an edge of $\Gamma$. Then $\Gamma$ is isomorphic to a coset graph $\operatorname{Cos}\left(G, G_{v}, G_{v} g G_{v}\right)$, where $g$ is a 2-element in $G$ such that $G_{u v}^{g}=G_{u v}, g^{2} \in G_{v}$ and $\left\langle G_{v}, g\right\rangle=G$. Moreover, $\Gamma$ has valency $\left|G_{v}: G_{v} \cap G_{v}^{g}\right|$.

In Proposition 2.5, the 2-element $g$ is called feasible to $G$ and $G_{v}$. Feasible $g$ can be found by MAGMA [1] when the order $|G|$ is not too large, and for convenience, an example of computer program to find feasible $g$ is given as an appendix at the end of the paper, which will be used in Section 3 frequently. 


\section{Proof of Theorem 1.1}

In this section, we always assume that $G$ is a non-abelian simple group. To prove Theorem 1.1, we need the following three lemmas.

Lemma 3.1 There is no connected tetravalent $A_{12}$-arc-transitive $A_{11}$-regular graph, and no connected tetravalent $M_{12}$-arc-transitive graph with stabilizer isomorphic to $S_{4}$.

Proof: Let $\Gamma$ be a connected tetravalent $T$-arc-transitive graph with $v \in V(\Gamma)$. By Proposition 2.5, $\Gamma=\operatorname{Cos}\left(T, T_{v}, T_{v} t T_{v}\right)$ for some feasible $t$, that is, $t$ is a 2-element such that $t^{2} \in T_{v},\left\langle T_{v}, t\right\rangle=T$ and $\left|T_{v}: T_{v} \cap T_{v}^{t}\right|=4$.

Let $T=A_{12}$ and let $\Gamma$ be $A_{11}$-regular. Then $\left|A_{11}\right|=|V(\Gamma)|$ and $\left|T_{v}\right|=|T| /\left|A_{11}\right|=12$. By Proposition 2.1, $T_{v} \cong A_{4}$. By MAGMA [1], $T_{v}$ has 12 conjugacy classes in $T$. Take a given $T_{v}$ in each conjugacy class, and computation shows that there is no such feasible $t$. It follows that there is no connected tetravalent $A_{12}$-arc-transitive $A_{11}$-regular graph.

Let $T=M_{12}$ and $T_{v} \cong S_{4}$. By MAGMA, $T_{v}$ has four conjugacy classes in $T$. Take a given $T_{v}$ in each conjugacy class, and computation shows that there is no such feasible $t$. Thus, there is no connected tetravalent $M_{12}$-arc-transitive graph with stabilizer isomorphic to $S_{4}$.

The radical of a group is defined as its largest soluble normal subgroup.

Lemma 3.2 Let $\Gamma$ be a connected tetravalent $G$-vertex-transitive graph and let $X$ be a 2-arc-transitive subgroup of Aut $(\Gamma)$ containing $G$. If $X$ has trivial radical, then either $G \unlhd X$, or $X$ has a normal arc-transitive non-abelian simple subgroup $T$ such that $G \leq T$ and $(G, T)=\left(M_{11}, M_{12}\right)$ or $\left(A_{n-1}, A_{n}\right)$ with $n \geq 8$ and $n \mid 2^{4} \cdot 3^{6}$. Moreover, if $\Gamma$ is $G$-regular then $(G, T)$ is listed in Table 1 .

Proof: Let $N$ be a minimal normal subgroup of $X$. Since $X$ has trivial radical, $N=T^{s}$ for a positive integer $s$ and a non-abelian simple group $T$. Clearly, $N G \leq X$. Since $\Gamma$ is $G$-vertex-transitive, by the Frattini argument we have $X=G X_{v}$ for $v \in V(\Gamma)$, and hence $|X|=|G|\left|X_{v}\right| /\left|G_{v}\right|$. Since $|N G|=|N||G| /|N \cap G|$, we have $|N| /|N \cap G||| X_{v}|/| G_{v} \mid$, and by Proposition [2.1, $|N| /|N \cap G| \mid 2^{4} \cdot 3^{6}$.

The simplicity of $G$ implies that $N \cap G=1$ or $G$. If $N \cap G=1$ then $|N|=|N| /|N \cap G| \mid$ $2^{4} \cdot 3^{6}$, which is impossible because $N$ is insoluble. Thus, $N \cap G=G$, that is, $G \leq N$, and $|N| /|G| \mid 2^{4} \cdot 3^{6}$. Since $T \unlhd N$, we have $T \cap G=1$ or $G$. If $T \cap G=1$ then $|T|=|T| /|G \cap T|=|G T| /|G||| N|/| G \mid$, which also is impossible because $|N| /|G| \mid 2^{4} \cdot 3^{6}$. Thus, $G \cap T=G$, that is, $G \leq T$. Since $|N| /|G|=|T|^{s-1}|T: G| \mid 2^{4} \cdot 3^{6}$, we have $s=1$. It follows that $G \leq T \unlhd X$ and $|T: G|=|T| /|G| \mid 2^{4} \cdot 3^{6}$.

If $G=T$ then $G \unlhd X$ and we are done. Now we may assume that $G$ is a proper subgroup of $T$. In particular, $G \notin X$. By Proposition 2.3, $(G, T)$ is listed in Table 2, Since $G \leq T \triangleleft X, \Gamma$ is $T$-vertex-transitive, and Proposition 2.2 implies that $\Gamma$ is $T$ arc-transitive. By Proposition 2.5. $\Gamma=\operatorname{Cos}\left(T, T_{v}, T_{v} t T_{v}\right)$ for some feasible $t$. Note that $|T| /|G|=|V(\Gamma)|\left|T_{v}\right| /\left(|V(\Gamma)|\left|G_{v}\right|\right)=\left|T_{v}\right| /\left|G_{v}\right|$. 
Suppose $(G, T)=\left(\operatorname{PSL}(2,13), G_{2}(3)\right)$. Then $\left|T_{v}\right| /\left|G_{v}\right|=|T| /|G|=2^{4} \cdot 3^{5}|| T_{v} \mid$. By Proposition 2.1, $T_{v} \cong \mathbb{Z}_{3}^{5} \rtimes \mathrm{GL}(2,3)$, and $|V(\Gamma)|=|T| /\left|T_{v}\right|=364$. However, there is no connected tetravalent 2-arc-transitive graph of order 364 by [14, Table 2], a contradiction.

Suppose $(G, T)=(\operatorname{PSL}(3,4), \operatorname{PSU}(4,3))$ or $\left(A_{7}, \operatorname{PSU}(4,3)\right)$. Then $3^{4}|| T_{v} \mid$ because $\left|T_{v}\right| /\left|G_{v}\right|=|T| /|G|$. By Proposition 2.1, $T_{v} \cong \mathbb{Z}_{3}^{5} \rtimes \operatorname{GL}(2,3)$, and by MAGMA, PSU $(4,3)$ has no subgroup isomorphic to $\mathbb{Z}_{3}^{5} \rtimes \mathrm{GL}(2,3)$, a contradiction.

Suppose $(G, T)=\left(A_{6}, \operatorname{PSp}(4,3)\right)$. Then $\left|T_{v}\right| /\left|G_{v}\right|=|T| /|G|=2^{3} \cdot 3^{2}|| T_{v} \mid$. Since $|T|=2^{6} \cdot 3^{4} \cdot 5$, Proposition 2.1 implies that $T_{v} \cong \mathbb{Z}_{3} \rtimes S_{4}, S_{3} \times S_{4}$ or $\operatorname{AGL}(2,3)$, and by MAGMA, PSp $(4,3)$ has no subgroup isomorphic to $\mathbb{Z}_{3} \rtimes S_{4}, S_{3} \times S_{4}$ or $\operatorname{AGL}(2,3)$, a contradiction.

Suppose $(G, T)=(\operatorname{PSL}(2,7), \operatorname{PSU}(3,3))$. Then $\left|T_{v}\right| /\left|G_{v}\right|=|T| /|G|=2^{2} \cdot 3^{2}|| T_{v} \mid$. Since $|T|=2^{5} \cdot 3^{2} \cdot 7$, Proposition 2.1 implies that $T_{v} \cong \mathbb{Z}_{3} \times A_{4}, \mathbb{Z}_{3} \rtimes S_{4}$ or $S_{3} \times S_{4}$, and by MAGMA, $\operatorname{PSU}(3,3)$ has no subgroup isomorphic to $\mathbb{Z}_{3} \times A_{4}, \mathbb{Z}_{3} \rtimes S_{4}$ or $S_{3} \times S_{4}$, a contradiction.

Suppose $(G, T)=\left(\operatorname{PSL}(2,11), M_{11}\right)$. Then $\left|T_{v}\right| /\left|G_{v}\right|=|T| /|G|=2^{2} \cdot 3|| T_{v} \mid$. Since $|T|=2^{4} \cdot 3^{2} \cdot 5 \cdot 7$, Proposition 2.1 implies that $T_{v} \cong A_{4}, S_{4}, \mathbb{Z}_{3} \times A_{4}, \mathbb{Z}_{3} \rtimes S_{4}$ or $S_{3} \times S_{4}$. By MAGMA, $M_{11}$ has no subgroup isomorphic to $\mathbb{Z}_{3} \times A_{4}, \mathbb{Z}_{3} \rtimes S_{4}$ or $S_{3} \times S_{4}$, and if $T_{v} \cong A_{4}$ or $S_{4}$ then $T_{v}$ has one conjugacy class, respectively. By taking a given $T_{v}$ in each conjugacy class, computation shows that there is no feasible $t$, a contradiction.

Suppose $(G, T)=\left(A_{7}, A_{9}\right)$. Then $\left|T_{v}\right| /\left|G_{v}\right|=|T| /|G|=2^{3} \cdot 3^{2}|| T_{v} \mid$. Since $3^{5} \nmid\left|A_{9}\right|$, Proposition 2.1 implies that $T_{v} \cong \mathbb{Z}_{3} \rtimes S_{4}, S_{3} \times S_{4}$ or AGL(2,3). By MAGMA, $A_{9}$ has no subgroup isomorphic to $\operatorname{AGL}(2,3)$ and if $T_{v} \cong \mathbb{Z}_{3} \rtimes S_{4}$ or $S_{3} \times S_{4}$ then $T_{v}$ has two conjugacy classes, respectively. By taking a given $T_{v}$ in each conjugacy class, computation shows that there is no feasible $t$, a contradiction.

Suppose $(G, T)=\left(M_{23}, M_{24}\right)$. Then $\left|T_{v}\right| /\left|G_{v}\right|=|T| /|G|=2^{3} \cdot 3$ and $2^{3} \cdot 3|| T_{v} \mid$. Since $3^{4} \nmid\left|M_{24}\right|$, Proposition 2.1 implies that $T_{v} \cong S_{4}, \mathbb{Z}_{3} \rtimes S_{4}, S_{3} \times S_{4}$ or $\operatorname{AGL}(2,3)$. By MAGMA, if $T_{v} \cong S_{4}$ then $T_{v}$ has 19 conjugacy classes, if $T_{v} \cong \operatorname{AGL}(2,3)$ then $T_{v}$ has one conjugacy class, and if $T_{v} \cong \mathbb{Z}_{3} \rtimes S_{4}$ or $S_{3} \times S_{4}$ then $T_{v}$ has four conjugacy classes, respectively. By taking a given $T_{v}$ in each conjugacy class, computation shows that there is no feasible $t$, a contradiction.

Suppose $(G, T)=\left(A_{8}, \operatorname{PSp}(6,2)\right)$. Then $\left|T_{v}\right| /\left|G_{v}\right|=|T| /|G|=2^{3} \cdot 3^{2}|| T_{v} \mid$. Since $3^{5} \nmid|\operatorname{PSp}(6,2)|$, Proposition 2.1 implies $T_{v} \cong \mathbb{Z}_{3} \rtimes S_{4}, S_{3} \times S_{4}$ or AGL(2,3). By MAGMA, $\operatorname{PSp}(6,2)$ has no subgroup isomorphic to $\operatorname{AGL}(2,3)$. If $T_{v} \cong \mathbb{Z}_{3} \rtimes S_{4}$ then $T_{v}$ has four conjugacy classes, and if $T_{v} \cong S_{3} \times S_{4}$ then $T_{v}$ has eight conjugacy classes. By taking a given $T_{v}$ in each conjugacy class, computation shows that there is no feasible $t$, a contradiction.

Suppose $(G, T)=\left(\operatorname{PSL}(2,11), M_{12}\right)$. Then $\left|T_{v}\right| /\left|G_{v}\right|=|T| /|G|=2^{4} \cdot 3^{2}|| T_{v} \mid$. Since $|T|=2^{6} \cdot 3^{3} \cdot 5 \cdot 11$, Proposition 2.1 implies that $T_{v} \cong S_{3} \times S_{4}$ or $\operatorname{AGL}(2,3)$. By MAGMA, $M_{12}$ has no subgroup isomorphic to $S_{3} \times S_{4}$ and if $T_{v} \cong \operatorname{AGL}(2,3)$ then $T_{v}$ has two conjugacy classes. By taking a given $T_{v}$ in each conjugacy class, computation shows that there is no feasible $t$, a contradiction.

Suppose $(G, T)=\left(A_{5}, A_{6}\right)$ Then $\left|T_{v}\right| /\left|G_{v}\right|=|T| /|G|=2 \cdot 3|| T_{v} \mid$. By Atlas [2, pp.4] and Proposition 2.1, $T_{v} \cong A_{4}$ or $S_{4}$, and by MAGMA, $T_{v}$ has two conjugacy classes, respectively. By taking a given $T_{v}$ in each conjugacy class, computation shows that there is no feasible $t$, a contradiction. 
By the above contradictions, $(G, T) \neq\left(\operatorname{PSL}(2,13), G_{2}(3)\right),(\operatorname{PSL}(3,4), \operatorname{PSU}(4,3)),\left(A_{7}\right.$, $\operatorname{PSU}(4,3)),\left(A_{6},(\operatorname{PSp}(4,3)),(\operatorname{PSL}(2,7), \operatorname{PSU}(3,3)),\left(\operatorname{PSL}(2,11), M_{11}\right),\left(A_{7}, A_{9}\right),\left(M_{23}\right.\right.$, $\left.M_{24}\right),\left(A_{8}, \operatorname{PSp}(6,2)\right),\left(\operatorname{PSL}(2,11), M_{12}\right)$ or $\left(A_{5}, A_{6}\right)$. Deleting the above impossible pairs from Table 2, we have $(G, T)=\left(M_{11}, M_{12}\right)$ or $\left(A_{n-1}, A_{n}\right)$ with $n \geq 8$ and $n \mid 2^{4} \cdot 3^{6}$ because $|T| /|G| \mid 2^{4} \cdot 3^{6}$. To finish the proof, let $\Gamma$ be $G$-regular. Then $G_{v}=1$ and $\left|T_{v}\right|=\left|T_{v}\right| /\left|G_{v}\right|=|T| /|G|$. By Proposition 2.1. $(G, T)=\left(M_{11}, M_{12}\right)$ or $\left(A_{n-1}, A_{n}\right)$ with $n=2^{2} \cdot 3,2^{3} \cdot 3,2^{2} \cdot 3^{2}, 2^{3} \cdot 3^{2}, 2^{4} \cdot 3^{2}, 2^{4} \cdot 3^{3}$ or $2^{4} \cdot 3^{6}$, and by Lemma 3.1 , $(G, T) \neq\left(A_{11}, A_{12}\right)$. It follows that $(G, T)$ is listed in Table 1 .

Remark: Let $(G, T)=\left(M_{11}, M_{12}\right)$. Then there is a unique connected tetravalent $T$-arctransitive $G$-regular graph $\Gamma$, and $\operatorname{Aut}(\Gamma) \cong M_{12} \rtimes \mathbb{Z}_{6}$ has non-trivial radical $\mathbb{Z}_{3}$. These facts can be checked by MAGMA. In fact, since $\left|T_{v}\right|=\left|T_{v}\right| /\left|G_{v}\right|=|T| /|G|=2^{2} \cdot 3$, Proposition 2.1 implies that $T_{v} \cong A_{4}$. By Proposition 2.5. $\Gamma=\operatorname{Cos}\left(T, T_{v}, T_{v} t T_{v}\right)$ for some feasible $t$, and by MAGMA, computation shows that $T_{v}$ has four conjugacy classes in $T$. Take a given $T_{v}$ in each conjugacy class: for two conjugacy classes there is no feasible $t$, and for the other two conjugacy classes, one has 24 feasible $t$ but all corresponding graphs $\Gamma$ are not $M_{11}$-vertex-transitive, and the other has 12 feasible $t$, of which the corresponding graphs $\Gamma$ are isomorphic to each other and $\operatorname{Aut}(\Gamma)=M_{12} \rtimes \mathbb{Z}_{6}$ with radical $\mathbb{Z}_{3}$.

Lemma 3.3 Let $\Gamma$ be a connected tetravalent 2-arc-transitive $G$-regular graph and let Aut $(\Gamma)$ have non-trivial radical $R$ with at least three orbits on $V(\Gamma)$. Assume $R G=R \times G$. Then $G \unlhd \operatorname{Aut}(\Gamma)$ or $\operatorname{Aut}(\Gamma)$ contains a normal arc-transitive non-abelian simple subgroup $T$ such that $G \leq T$ and $(G, T)$ is listed in Table 1 .

Proof: Set $A=\operatorname{Aut}(\Gamma)$ and $B=R G=R \times G$. Then $G$ is characteristic in $B$. To finish the proof, we may assume $G \not A$ and aim to show that $A$ contains a normal arc-transitive non-abelian simple subgroup $T$ such that $G \leq T$ and $(G, T)$ is listed in Table 1 .

Since $R \neq 1$ has at least three orbits, by Proposition 2.2 the quotient graph $\Gamma_{R}$ is a connected tetravalent $(A / R, 2)$-arc-transitive graph with $A / R \leq \operatorname{Aut}\left(\Gamma_{R}\right)$, and $\Gamma_{R}$ is $B / R$-vertex-transitive. Since $G \not A$ and $G$ is characteristic in $B$, we have $B \not A$ and $G \cong B / R \not A / R$. Furthermore, $A / R$ has trivial radical as $R$ is the radical of $A$. By Lemma 3.2, $A / R$ has a normal arc-transitive subgroup $I / R$ such that $B / R \leq I / R$ and $(B / R, I / R) \cong(G, T)=\left(M_{11}, M_{12}\right)$ or $\left(A_{n-1}, A_{n}\right)$ with $n \geq 8$ and $n \mid 2^{4} \cdot 3^{6}$.

Note that $I \unlhd A$. Since $A$ is 2-arc-transitive, $I$ is arc-transitive. Let $C=C_{I}(R)$. Then $C \unlhd I$ and $C \cap R \leq Z(C)$. Since $B=R \times G \leq I$, we have $G \leq C$, and since $C / C \cap R \cong$ $C R / R \unlhd I / R \cong T$, we have $C \cap R=Z(C), C / Z(C) \cong T$ and $I=C R$. Furthermore, $C^{\prime} / C^{\prime} \cap Z(C) \cong C^{\prime} Z(C) / Z(C)=(C / Z(C))^{\prime}=C / Z(C) \cong T$. Thus, $Z\left(C^{\prime}\right)=C^{\prime} \cap Z(C)$, $C=C^{\prime} Z(C)$ and $C^{\prime} / Z\left(C^{\prime}\right) \cong T$. It follows that $C^{\prime}=\left(C^{\prime} Z(C)\right)^{\prime}=C^{\prime \prime}$, and so $C^{\prime}$ is a covering group of $T$. Since $C / C^{\prime}$ is abelian, $G \leq C^{\prime}$.

Recall that $(G, T)=\left(M_{11}, M_{12}\right)$ or $\left(A_{n-1}, A_{n}\right)$ with $n \geq 8$ and $n \mid 2^{4} \cdot 3^{6}$. By [9, Theorem 5.1.4] and [2, pp.31], the Schur multiplier $\operatorname{Mult}\left(A_{n}\right)=\mathbb{Z}_{2}$ for $n \geq 8$ and $\operatorname{Mult}\left(M_{12}\right)=\mathbb{Z}_{2}$. Then $T$ has a unique double cover, denoted by $2 . T$.

Suppose $Z\left(C^{\prime}\right)=\mathbb{Z}_{2}$. Then $C^{\prime}$ is the unique double cover of $T$, that is, $C^{\prime}=2 . T$. Let $(G, T)=\left(M_{11}, M_{12}\right)$. Then $\left|C^{\prime}\right|=2\left|M_{12}\right|$, and since $\Gamma$ is $G$-regular, $\left|C_{v}^{\prime}\right|=24$ for 
$v \in V(\Gamma)$. In particular, 3||$I_{v} \mid$, and $I$ is 2-arc-transitive by the arc-transitivity of $I$. Since $C \unlhd I$ and $C^{\prime}$ is characteristic in $C$, we have $C^{\prime} \unlhd I$ and so $C^{\prime}$ is arc-transitive. Similarly, $C^{\prime}$ is 2-arc-transitive as 3||$C_{v}^{\prime} \mid$, and by Proposition 2.1 , $C_{v}^{\prime} \cong S_{4}$. The quotient graph $\Gamma_{Z\left(C^{\prime}\right)}$ is a connected tetravalent $\left(C^{\prime} / Z\left(C^{\prime}\right), 2\right)$-arc-transitive graph with stabilizer isomorphic to $C_{v}^{\prime}$. Since $C_{v}^{\prime} \cong S_{4}$ and $C^{\prime} / Z\left(C^{\prime}\right) \cong M_{12}$, this is impossible by Lemma 3.1] Now let $(G, T)=\left(A_{n-1}, A_{n}\right)$ with $n \geq 8$. Then $C^{\prime}=2$. $A_{n}$. Since $Z\left(C^{\prime}\right) \unlhd I$ and $I / R \cong T$, we have $Z\left(C^{\prime}\right) \leq R$, and since $B=G \times R$, we have $G \times Z\left(C^{\prime}\right) \leq C^{\prime}$. Then $G \times Z\left(C^{\prime}\right)$ is a subgroup of index $n$ of $C^{\prime}$ isomorphic to $A_{n-1} \times \mathbb{Z}_{2}$. This is impossible by Proposition 2.4.

Thus, $Z\left(C^{\prime}\right)=1$. It follows that $C^{\prime} \cong T$ and $G \leq C^{\prime} \unlhd I$. Since $|I|=|I / R||R|=$ $|T||R|=\left|C^{\prime}\right||R|$ and $C^{\prime} \cap R=1$, we have $I=C^{\prime} \times R$ because $C^{\prime} \unlhd I$, and so $C^{\prime}$ is characteristic in $I$. Since $I \unlhd A$ and $A$ is 2-arc-transitive, $A$ has a normal arc-transitive non-abelian simple subgroup $C^{\prime} \cong T$ containing $G$ and $(G, T)=\left(M_{11}, M_{12}\right)$ or $\left(A_{n-1}, A_{n}\right)$ with $n \geq 8$ and $n \mid 2^{4} \cdot 3^{6}$. Since $\Gamma$ is $G$-regular, $\left|C_{v}^{\prime}\right|=\left|C_{v}^{\prime}\right| /\left|G_{v}\right|=\left|C^{\prime}\right| /|G|=|T: G|$, and by Proposition 2.1, $(G, T)=\left(M_{11}, M_{12}\right)$ or $\left(A_{n-1}, A_{n}\right)$ with $n=2^{2} \cdot 3,2^{3} \cdot 3,2^{2} \cdot 3^{2}$, $2^{3} \cdot 3^{2}, 2^{4} \cdot 3^{2}, 2^{4} \cdot 3^{3}$ or $2^{4} \cdot 3^{6}$. Furthermore, $(G, T) \neq\left(A_{11}, A_{12}\right)$ by Lemma 3.1. It follows that $(G, T)$ is listed in Table 1 .

Now, we are ready to prove Theorem 1.1 .

The proof of Theorem 1.1: Let $G$ be a non-abelian simple group and $\Gamma$ a connected tetravalent 2-arc-transitive $G$-regular graph with $v \in V(\Gamma)$. Then $G_{v}=1$ and $|G|=$ $|V(\Gamma)|$. Set $A=\operatorname{Aut}(\Gamma)$ and let $R$ be the radical of $A$. By Lemma 3.2, the theorem is true for $R=1$. Thus, we may assume $R \neq 1$.

Set $B=R G$. Then $G \cap R=1$ and so $|B|=|R||G|$. Since $\Gamma$ is $G$-regular, $B=G B_{v}$ and $|B|=|G|\left|B_{v}\right|$. It follows that $|R|=\left|B_{v}\right|$, and by Proposition 2.1, $|R| \mid 2^{4} \cdot 3^{6}$.

Suppose that $R$ has one or two orbits on $V(\Gamma)$. Since $\Gamma$ is a connected tetravalent $G$-regular graph, $|R|=\left|R_{v}\right|\left|v^{R}\right|=\left|R_{v}\right||G|$ or $\left|R_{v}\right||G| / 2$. Since $|R| \mid 2^{4} \cdot 3^{6}$, the non-abelian simple group $G$ is a $\{2,3\}$-group, which is impossible.

Thus, $R$ has at least three orbits. If $B=R \times G$ then the theorem is true by Lemma 3.3. Now assume $B \neq R \times G$, and to finish the proof, we aim to derive contradictions.

Since $|R| \mid 2^{4} \cdot 3^{6}$, we may write $|R|=2^{m} \cdot 3^{k}$, where $0 \leq m \leq 4$ and $0 \leq k \leq 6$. Since $R$ is soluble, there exists a series of principle subgroups of $B$ :

$$
B>R=R_{s}>\cdots>R_{1}>R_{0}=1
$$

such that $R_{i} \unlhd B$ and $R_{i+1} / R_{i}$ is an elementary abelian $r$-group with $0 \leq i \leq s-1$ and $r=2$ or 3 . Let $\left|R_{i+1} / R_{i}\right|=r^{\ell_{i}}$. Then $\ell_{i} \leq m \leq 4$ if $r=2$, and $\ell_{i} \leq k \leq 6$ if $r=3$. Note that $G \leq B$ has a natural action on $R_{i+1} / R_{i}$ by conjugation.

Since $B \neq R \times G$, there exists $0 \leq j \leq s-1$ such that $G R_{j}=G \times R_{j}$ and $G R_{j+1} \neq$ $G \times R_{j+1}$. If $G$ acts trivially on $R_{j+1} / R_{j}$, then $\left[G R_{j} / R_{j}, R_{j+1} / R_{j}\right]=1$. Since $G R_{j} / R_{j} \cong G$ is simple, $\left(G R_{j} / R_{j}\right) \cap\left(R_{j+1} / R_{j}\right)=1$, and since $\left|G R_{j+1} / R_{j}\right|=\left|G R_{j+1} / R_{j+1}\right|\left|R_{j+1} / R_{j}\right|=$ $|G|\left|R_{j+1} / R_{j}\right|=\left|G R_{j} / R_{j}\right|\left|R_{j+1} / R_{j}\right|$, we have $G R_{j+1} / R_{j}=G R_{j} / R_{j} \times R_{j+1} / R_{j}$. In particular, $G R_{j} \unlhd G R_{j+1}$ and so $G \unlhd G R_{j+1}$ because $G R_{j}=G \times R_{j}$ implies that $G$ is characteristic in $G R_{j}$. It follows that $G R_{j+1}=G \times R_{j+1}$, a contradiction. Thus, $G$ acts non-trivially on $R_{j+1} / R_{j}$, and the simplicity of $G$ implies that the action is faithful. Since $R_{j+1} / R_{j}$ is an 
elementary abelian group of order $r^{\ell_{j}}$, we have $G \leq \mathrm{GL}\left(\ell_{j}, r\right)$, where $\ell_{j} \leq m \leq 4$ if $r=2$ and $\ell_{j} \leq k \leq 6$ if $r=3$.

By Proposition 2.2, $R$ is semiregular on $V(\Gamma)$, and the quotient graph $\Gamma_{R}$ is a connected tetravalent $(A / R, 2)$-arc-transitive graph with $A / R \leq \operatorname{Aut}\left(\Gamma_{R}\right)$. Moreover, $\Gamma_{R}$ is $B / R$-vertex-transitive, and $|B / R|=\left|V\left(\Gamma_{R}\right)\right|\left|(B / R)_{\alpha}\right|$ for $\alpha \in V\left(\Gamma_{R}\right)$. Since $\Gamma$ is $G$ regular, $\left|V\left(\Gamma_{R}\right)\right|=|V(\Gamma)| /|R|=|G| /|R|$, and since $B / R \cong G$, we have $|G|=|B / R|=$ $\left|V\left(\Gamma_{R}\right)\right|\left|(B / R)_{\alpha}\right|=|G| /|R| \cdot\left|(B / R)_{\alpha}\right|$. It follows that $|R||| G \mid$ and $\left|(B / R)_{\alpha}\right|=|R|$.

Since $R$ is the largest normal soluble subgroup of $A, A / R$ has trivial radical, and since $B / R \cong G$, Lemma 3.2 implies that either $B / R \unlhd A / R$, or $A / R$ has a normal arctransitive subgroup $I / R$ such that $B / R \leq I / R$ and $(B / R, I / R) \cong(G, T)=\left(M_{11}, M_{12}\right)$ or $\left(A_{n-1}, A_{n}\right)$ with $n \geq 8$ and $n \mid 2^{4} \cdot 3^{6}$.

Case 1: $B / R \unlhd A / R$.

In this case, $B \unlhd A$ and $B_{v} \unlhd A_{v}$. Since $A$ is 2-arc-transitive, $B$ is arc-transitive and $\Gamma_{R}$ is $B / R$-arc-transitive. Thus, 4||$B_{v} \mid$ and $\Gamma_{R} \cong \operatorname{Cos}\left(B / R,(B / R)_{\alpha},(B / R)_{\alpha} g(B / R)_{\alpha}\right)$ for some feasible $g$. Recall that $|R|=\left|B_{v}\right|=\left|(B / R)_{\alpha}\right|=2^{m} \cdot 3^{k},|R||| G \mid$ and $G \leq \operatorname{GL}\left(\ell_{j}, r\right)$, where $\ell_{j} \leq m \leq 4$ if $r=2$ and $\ell_{j} \leq k \leq 6$ if $r=3$.

By Proposition 2.1, $A_{v} \cong A_{4}, \mathbb{Z}_{3} \times A_{4}, S_{4}, \mathbb{Z}_{3} \rtimes S_{4}, S_{3} \times S_{4}, \operatorname{AGL}(2,3)$ or $\mathbb{Z}_{3}^{5} \rtimes \operatorname{GL}(2,3)$.

Suppose $A_{v} \cong A_{4}, \mathbb{Z}_{3} \times A_{4}, S_{4}, \mathbb{Z}_{3} \rtimes S_{4}$ or $S_{3} \times S_{4}$. Since $|R|=\left|B_{v}\right|$, we have $|R|=2^{m} \cdot 3^{k}$, where $m \leq 4$ and $k \leq 2$. Since $\operatorname{GL}(2,2)$ and $\operatorname{GL}(2,3)$ are soluble and $G \leq \operatorname{GL}\left(\ell_{j}, r\right)$, we have either $m=4$ and $G \leq \operatorname{GL}(4,2)=\operatorname{PSL}(4,2)$, or $m=3$ and $G=\operatorname{PSL}(3,2)$.

If $m=4$ and $G \leq \operatorname{PSL}(4,2)$ then $2^{4}|| B_{v} \mid$ and $A_{v}=S_{3} \times S_{4}$. Since $B_{v} \unlhd A_{v}$, we have $B_{v}=S_{3} \times S_{4}$, and by Proposition $\left[2.1\right.$, $\left|(B / R)_{\alpha}\right|=\left|B_{v}\right|=2^{4} \cdot 3^{2}$ implies that $(B / R)_{\alpha}=S_{3} \times S_{4}$. This is impossible because $B / R \cong G \leq \operatorname{PSL}(4,2)$ and $\operatorname{PSL}(4,2)$ has no subgroup isomorphic to $S_{3} \times S_{4}$ by MAGMA. If $m=3$ and $G=\operatorname{PSL}(3,2)$ then $A_{v}=S_{4}$, $\mathbb{Z}_{3} \rtimes S_{4}$ or $S_{3} \times S_{4}$. Since $B_{v} \unlhd A_{v}$ and $2^{3}|| B_{v} \mid$, we have $\left|(B / R)_{\alpha}\right|=\left|B_{v}\right|=2^{3} \cdot 3,2^{3} \cdot 3^{2}, 2^{4} \cdot 3$ or $2^{4} \cdot 3^{2}$, and by Proposition [2.1, $(B / R)_{\alpha} \cong S_{4}, \mathbb{Z}_{3} \rtimes S_{4}$ or $S_{3} \times S_{4}$. By Atlas [2, pp.3], $(B / R)_{\alpha} \cong S_{4}$ as $B / R \cong \operatorname{PSL}(3,2)$. By MAGMA, $(B / R)_{\alpha}$ has two conjugacy classes in $B / R$, and for both classes, there are no feasible $g$, a contradiction.

Suppose $A_{v} \cong \operatorname{AGL}(2,3)$. Since 4||$B_{v} \mid$ and $B_{v} \triangleleft A_{v}$, by MAGMA $B_{v}=A_{v}$, and so $|R|=2^{4} \cdot 3^{3}$. Since $3^{3} \nmid|\mathrm{GL}(4,2)|$, we have $G \leq \mathrm{GL}(3,3)$, and by MAGMA, $G=$ $\operatorname{PSL}(3,3)$. Since $\left|(B / R)_{\alpha}\right|=|R|=2^{4} \cdot 3^{3}$, by Proposition $\left[2.1(B / R)_{\alpha} \cong \operatorname{AGL}(2,3)\right.$. By MAGMA, there are two conjugacy classes isomorphic to $\operatorname{AGL}(2,3)$ in $\operatorname{PSL}(3,3)$, and for each conjugacy class, there is no feasible $g$, a contradiction.

Suppose $A_{v} \cong \mathbb{Z}_{3}^{5} \rtimes \mathrm{GL}(2,3)$. Since 4||$B_{v} \mid$ and $B_{v} \unlhd A_{v}$, by the Remark of Proposition 2.1. $B_{v}=A_{v}$ and $|R|=\left|(B / R)_{\alpha}\right|=2^{4} \cdot 3^{6}$. Thus, $B$ is 7 -arc-transitive and $G \cong B / R$ has a subgroup of order $2^{4} \cdot 3^{6}$. Since $2^{4} \cdot 3^{6} \nmid|\mathrm{GL}(4,2)|$, we have $G \not \leq \operatorname{GL}(4,2)$. Thus, $G \leq \mathrm{GL}\left(\ell_{j}, 3\right)$ with $\ell_{j} \leq k \leq 6$. By MAGMA, for $1 \leq k \leq 4, \mathrm{GL}(k, 3)$ have no simple subgroup $G$ with a subgroup of order $2^{4} \cdot 3^{6}$. It follows that $\ell_{j}=5$ or 6 with $r=3$, and hence $\left|R_{j+1} / R_{j}\right|=3^{5}$ or $3^{6}$, where $0 \leq j \leq s-1$.

Let $j \neq s-1$. Then $\left|R / R_{s-1}\right|=3$ or $2^{t}$ for $1 \leq t \leq 4$, and so $\left|R_{s-1}\right|=2^{4} \cdot 3^{5}$ or $2^{4-t} \cdot 3^{6}$. Since $G$ is simple and $G \not \leq \mathrm{GL}(4,2), G$ acts trivially on $R / R_{s-1}$ by conjugation, implying $\left[G R_{s-1} / R_{s-1}, R / R_{s-1}\right]=1$. It follows that $G R / R_{s-1}=G R_{s-1} / R_{s-1} \times R / R_{s-1}$ and hence $G R_{s-1} \unlhd G R=B$. Since $B$ is 7 -arc-transitive, $G R_{s-1}$ is arc-transitive, and since $G$ is 
regular, $G R_{s-1}$ has a stabilizer of order $2^{4} \cdot 3^{5}$ or $2^{4-t} \cdot 3^{6}$, contrary to Proposition 2.1.

Let $j=s-1$. Then $\left|R / R_{s-1}\right|=3^{5}$ or $3^{6}$, and $\left|R_{s-1}\right|=2^{4} \cdot 3$ or $2^{4}$. Furthermore, $G R_{s-1}=G \times R_{s-1}$ and $B=G R \neq G \times R$. Clearly, $R=R_{s-1} P$ for a Sylow 3-subgroup $P$ of $R$. Let $C=C_{B}\left(R_{s-1}\right)$. Then $C \unlhd B$ and $G \leq C$. If $G=C$ then $G \triangleleft B$ and so $B=G \times R$, a contradiction. Thus, $G$ is a proper subgroup of $C$, so that $C_{v} \neq 1$. Since $B$ is 7-arc-transitive, $C$ is arc-transitive and by the Remark of Proposition 2.1. $C_{v}=B_{v}$, that is, $B=C$. In particular, $\left[P, R_{s-1}\right]=1$. Since $R=P R_{s-1}, P$ is normal in $R$ and so characteristic. This implies $P \unlhd B$ and so $G P \leq B=C$. It follows $\left[G P, R_{s-1}\right]=1$, and since $B=G R=(G P) R_{s-1}$, we have $G P \unlhd B$. Since $B$ is 7 -arc-transitive, $G P$ is arc-transitive, forcing 4||$(G P)_{v} \mid$, and since $G$ is regular, $\left|(G P)_{v}\right|=|P|$ and thus 4||$P \mid$, contrary to the fact that $P$ is a Sylow 3-subgroup of $R$.

Case 2: $A / R$ has a normal arc-transitive subgroup $I / R$ such that $B / R \leq I / R$ and $(B / R, I / R) \cong(G, T)=\left(M_{11}, M_{12}\right)$ or $\left(A_{n-1}, A_{n}\right)$ with $n \geq 8$ and $n \mid 2^{4} \cdot 3^{6}$.

In this case, $I \unlhd A$ and $I$ is arc-transitive. Since $|T|=|I / R|=\left|V\left(\Gamma_{R}\right)\right|\left|(I / R)_{\alpha}\right|=$ $|G| /|R| \cdot\left|(I / R)_{\alpha}\right|$, we have $\left|(I / R)_{\alpha}\right|=|R||T| /|G|$. By Proposition 2.1, $|R||T| /|G|$ is a divisor of $2^{4} \cdot 3^{6}$. Recall that $|R|=\left|B_{v}\right|=\left|(B / R)_{\alpha}\right|=2^{m} \cdot 3^{k},|R||| G \mid$ and $G \leq \mathrm{GL}\left(\ell_{j}, r\right)$, where $\ell_{j} \leq m \leq 4$ if $r=2$ or $\ell_{j} \leq k \leq 6$ if $r=3$.

Suppose $(G, T)=\left(M_{11}, M_{12}\right)$. Then $|T| /|G|=2^{2} \cdot 3$. Since $|R||T| /|G| \mid 2^{4} \cdot 3^{6}$, we have $|R| \mid 2^{2} \cdot 3^{5}$, and then $|R||| G \mid$ implies that $|R| \mid 2^{2} \cdot 3^{3}$. Since $\operatorname{GL}(2,2)$ is soluble, we have $G \leq \mathrm{GL}(3,3)$, which is impossible because 11||$M_{11} \mid$ and $11 \nmid|\mathrm{GL}(3,3)|$.

Suppose $(G, T)=\left(A_{n-1}, A_{n}\right)$ with $n \geq 8$ and $n \mid 2^{4} \cdot 3^{6}$. If $n \geq 12$ then $5^{2}|| G \mid$, which is impossible because $G \leq \mathrm{GL}(4,2)$ or $\mathrm{GL}(6,3)$ but $5^{2} \nmid|\mathrm{GL}(6,3)|$ and $5^{2} \nmid \mathrm{GL}(4,2)$. It follows that $8 \leq n<12$, and since $n \mid 2^{4} \cdot 3^{6}$, we have $(G, T)=\left(A_{7}, A_{8}\right)$ or $\left(A_{8}, A_{9}\right)$.

Suppose $(G, T)=\left(A_{7}, A_{8}\right)$. Then $|T| /|G|=2^{3}$. Since $|R||T| /|G| \mid 2^{4} \cdot 3^{6}$, we have $|R| \mid 2 \cdot 3^{6}$, and $|R||| G \mid$ implies that $|R| \mid 2 \cdot 3^{2}$. Thus, $G \leq \mathrm{GL}(1,2)$ or $\operatorname{GL}(2,3)$, yielding that $G$ is soluble, a contradiction.

Suppose $(G, T)=\left(A_{8}, A_{9}\right)$. Then $|T| /|G|=3^{2}$. Since $|R||T| /|G| \mid 2^{4} \cdot 3^{6}$, we have $|R| \mid 2^{4} \cdot 3^{4}$ and $|R||| G \mid$ implies $|R| \mid 2^{4} \cdot 3^{2}$, that is, $|R|=2^{m} \cdot 3^{k}$ with $m \leq 4$ and $k \leq 2$. Since $\mathrm{GL}(2,3)$ is soluble, $G \leq \mathrm{GL}(4,2)$, and hence $G=\mathrm{GL}(4,2)$ and $m=4$ as $G=A_{8} \cong$ $\operatorname{GL}(4,2)$. Since $\left|(I / R)_{\alpha}\right|=|R||T| /|G|$, we have $2^{4} \cdot 3^{2}||(I / R)_{\alpha} \mid$, and since $|I / R|=\left|A_{9}\right|=$ $2^{6} \cdot 3^{4} \cdot 5 \cdot 7$, Proposition 2.1 implies that $(I / R)_{\alpha} \cong S_{3} \times S_{4}$ or $\operatorname{AGL}(2,3)$. By the arctransitivity of $I, \Gamma_{R}$ is $I / R$-arc-transitive, and so $\Gamma_{R}=\operatorname{Cos}\left(I / R,(I / R)_{\alpha},(I / R)_{\alpha} t(I / R)_{\alpha}\right)$ for some feasible $t$. By MAGMA, $I / R \cong A_{9}$ has no subgroup isomorphic to $\operatorname{AGL}(2,3)$ and $I / R$ has two conjugacy classes isomorphic to $S_{3} \times S_{4}$. By taking a given $(I / R)_{\alpha}$ in each conjugacy class, computation shows that there is no feasible $t$, a contradiction.

To end the paper, we give examples to show that the pair $(G, T)=\left(A_{23}, A_{24}\right)$ in Theorem 1.1 can happen.

Example 3.4 Let $G=A_{23}$ and $T=A_{24}$. Define $x, y, z, w, g \in T$ as following:

$$
\begin{aligned}
& x=(1,2)(3,7)(4,10)(5,13)(6,15)(8,12)(9,19)(11,18)(14,22)(16,20)(17,24)(21,23), \\
& y=(1,3)(2,7)(4,8)(5,9)(6,18)(10,12)(11,15)(13,19)(14,20)(16,22)(17,23)(21,24),
\end{aligned}
$$




$$
\begin{aligned}
& z=(1,4,6)(2,8,11)(3,12,15)(5,17,16)(7,10,18)(9,21,20)(13,23,14)(19,24,22), \\
& w=(1,5)(2,9)(3,13)(4,16)(6,17)(7,19)(8,20)(10,22)(11,21)(12,14)(15,23)(18,24), \\
& g_{1}=(1,5)(2,10)(3,14)(4,17)(6,16)(7,11)(8,18)(9,22)(12,13)(15,23)(19,21)(20,24), \\
& g_{2}=(1,5)(2,10)(3,8)(4,16)(6,15)(7,19)(9,22)(11,12)(13,20)(14,18)(17,24)(21,23), \\
& g_{3}=(1,5)(2,9)(3,13)(4,16)(6,15)(7,14)(8,20)(10,19)(11,17)(12,23)(18,22)(21,24), \\
& g_{4}=(1,2)(3,12)(4,8)(5,9)(6,10)(7,19)(11,23)(13,22)(14,18)(15,20)(16,21)(17,24) .
\end{aligned}
$$

By MAGAMA[1], $H=\langle x, y, z, w\rangle \cong S_{4}, T=\left\langle H, g_{i}\right\rangle,\left|H: H \cap H^{g_{i}}\right|=4(1 \leq i \leq 4)$ and $H$ is regular on $\{1,2, \cdots, 24\}$. Thus, $T=G H$ with $G \cap H=1$. Define $\Gamma_{i}=$ $\operatorname{Cos}\left(T, H, H g_{i} H\right)$ with $1 \leq i \leq 4$. Then $\Gamma_{i}$ is a connected tetravalent $(T, 2)$-arc-transitive $G$-regular graph, where $G$ and $T$ are viewed as groups of automorphisms of $\Gamma_{i}$ by right multiplication. By Theorem 1.1, $T \unlhd \operatorname{Aut}\left(\Gamma_{i}\right)$ with $1 \leq i \leq 4$. Again by MAGMA, $\operatorname{Aut}(T, H, H g H) \cong \tilde{H}$, where $\tilde{H}$ is the automorphism group of $T$ induced by conjugate of elements in $H$. Thus, $\operatorname{Aut}\left(\Gamma_{i}\right)=T(1 \leq i \leq 4)$ by [18, Lemma 2.10].

Acknowledgement: This work was supported by the National Natural Science Foundation of China $(11571035,11231008)$ and by the 111 Project of China (B16002). 


\section{Appendix}

Let $G$ be a finite group and $H \leq G$. Let $\Gamma=\operatorname{Cos}(G, H, H g H)$ be a connected tetravalent $G$-arc-transitive graph. Then $g$ can be chosen as a 2 -element such that $g^{2} \in H$, $\langle H, g\rangle=G,\left|H: H \cap H^{g}\right|=4$, and such a $g$ is called feasible. There are many places in the paper to compute feasible $g$ for given $G$ and $H$ and to compute their full automorphism groups of the corresponding coset graphs. Here we provide a computer program based on MAGMA language by taking $(G, H)=\left(M_{12}, A_{4}\right)$ as an example:

load "M12"; B:=Subgroups(G);

$\mathrm{PG}:=[] ; / /$ possible graphs

for $\mathrm{i}$ in $[1 . . \# \mathrm{~B}]$ do if IsIsomorphic(Alt(4),B[i]'subgroup) then $\mathrm{H}:=\mathrm{B}[\mathrm{i}]$ 'subgroup; print $\mathrm{i}$;

$\mathrm{D}:=[] ; / /$ feasible elements

for $g$ in $G$ do if IsDivisibleBy $\left(2^{6}\right.$, Order(g)) and $\operatorname{Order}\left(\operatorname{sub}\left\langle G \mid H, g^{2}\right\rangle\right)$ eq $\operatorname{Order}(H)$ and \# $(H * g * H)$ eq $\operatorname{Order}(H) * 4$ and $\operatorname{Order}(\operatorname{sub}\langle G \mid H, g\rangle)$ eq $\operatorname{Order}(G)$ then $\operatorname{Include}(\sim \mathrm{D}, \mathrm{g})$; end if; end for;

\#D;

if $\# \mathrm{D}$ ne 0 then for $\mathrm{j}$ in $[1 . . \# \mathrm{D}]$ do $\mathrm{c}:=\mathrm{D}[\mathrm{j}] ; \mathrm{HcH}:=\{\}$;

for $t$ in $H$ do for $h$ in $H$ do Include $(\sim \mathrm{HcH}, \mathrm{t} * \mathrm{c} * \mathrm{~h})$; end for; end for;

$\mathrm{Vj}:=\{\} ; \mathrm{Ej}:=\{\} ;$

for $t$ in $G$ do for $s$ in $\mathrm{HcH}$ do $\mathrm{T} 1:=\{\} ; \mathrm{T} 2:=\{\}$; for $h$ in $H$ do Include $(\sim \mathrm{T} 1, \mathrm{~h} * \mathrm{t})$; Include $(\sim \mathrm{T} 2, \mathrm{~h} * \mathrm{~s} * \mathrm{t})$; end for; Include $(\sim \mathrm{Vj}, \mathrm{T} 1)$; Include $(\sim \mathrm{Ej},\{\mathrm{T} 1, \mathrm{~T} 2\})$; end for; end for;

$\mathrm{PGj}:=\operatorname{Graph}\langle\mathrm{Vj} \mid \mathrm{Ej}\rangle ; \operatorname{Include}(\sim \mathrm{PG}, \mathrm{PGj})$;

end for; end if; end if; end for;

NPG:=[];// non-isomorphic possible graphs

$\mathrm{NPG}:=[\mathrm{PG}[1]]$

for $\mathrm{k}$ in $[1 . . \# \mathrm{PG}]$ do $\mathrm{p}:=0$; for $\mathrm{m}$ in $[1 . . \# \mathrm{NPG}]$ do if IsIsomorphic $(\mathrm{PG}[\mathrm{k}], \mathrm{NPG}[\mathrm{m}])$ then $\mathrm{p}:=\mathrm{p}+0$; else $\mathrm{p}:=\mathrm{p}+1$; end if; end for;

if $\mathrm{p}$ eq \#NPG then Include( NPG, PG[k]); end if; end for;

Graph:=[ ]; // $M_{12}$-arc-transitive $M_{11}$-regular graph

for $\mathrm{t}$ in $[1 . . \# \mathrm{NPG}]$ do $\mathrm{A}:=$ AutomorphismGroup (NPG[t]); S:=Subgroups(A);

for $\mathrm{n}$ in [1..\#S] do if IsTransitive(S[n]'subgroup) and Order(S[n]'subgroup) eq 7920 and IsSimple(S[n]'subgroup) then Include( Graph, NPG[t]);

print "We find a connected tetravalent $M_{12}$-arc-transitive $M_{11}$-regular graph";

print "The automorphism group A:" ; print A;

print "The radical of A:"; print Radical(A);

end if; end for; end for;

\#Graph; 


\section{References}

[1] Bosma W, Cannon C, Playoust C. The MAGMA algebra system I: The user language. J Symbolic Comput, 1997, 24: 235-265

[2] Conway J H, Curtis R T, Norton S P, Parker R A, Wilson R A. Atlas of Finite Group. London-New York: Oxford University Press, 1985

[3] Fang X G, Li C H, Xu M Y. On edge-transitive Cayley graphs of valency four. Europ J Combin, 2004, 25: 1107-1116

[4] Fang X G, Praeger C E. Finite two-arc transitive graphs admitting a Suzuki simple group. Comm Algebra, 1999, 27: 3727-3754

[5] Gardiner A, Praeger C E. On 4-valent symmetric graphs. Europ J Combin, 1994, 15: 375-381

[6] Godsil C D. On the full automorphism group of a graph. Combinatorica, 1981, 1: 243-256

[7] Guralnick R M. Subgroups of prime power index in a simple group. J Algebra, 1983, 81: $304-311$

[8] Huppert B. Eudiche Gruppen I. Berlin: Springer-Verlag, 1967

[9] Kleidman P, M. Liebeck M. The Subgroup Structure of the Finite Classical Groups. Cambridge: Cambridge University Press, 1990

[10] Li C H. Isomorphisms of finite Cayley graphs Ph.D. Thesis. The University of Western Australia, 1996

[11] Li X H, Xu M Y. The primitive permutation groups of degree $2^{a} \cdot 3^{b}$. Arch Math, 2006, 86: 385-391

[12] Lorimer P. Vertex-transitive graphs: symmetric graphs of prime valency. J Graph Theory, 1984, 8: 55-68

[13] Miller R C. The trivalent symmetric graphs of girth at most six. J Combin Theory Ser B, 1971, 10: 163-182

[14] Potočnik P. A list of 4-valent 2-arc-transitive graphs and finite faithful amalgams of index (4,2). Europ J Combin, 2009, 30: 1323-1336

[15] Praeger C E. An O'Nan-Scott theorem for finite quasiprimitive permutation groups and an application to 2-arc transitive graphs. J Lond Math Soc, 1993, 47: 227-239

[16] Sabidussi B O. Vertex-transitive graphs. Monash Math, 1964, 68: 426-438 
[17] Schur J. Über die Darstellung der endlichen Gruppen durch gebrochene lineare Substitutionen. J Reine Angew Math, 1904, 127: 20-50

[18] Wang Y, Feng Y Q, Zhou J X. Cayley digraphs of 2-genetic groups of odd prime-power order. J Combin Theory Ser A, 2016, 143: 88-106

[19] Wilson R A. The Finite Simple Groups. London: Springer-Verlag, 2009

[20] Xu M Y. Automorphism groups and isomorphisms of Cayley digraphs. Discrete Math, 1998, 182: 309-319

[21] Xu S J, Fang X G, Wang J, Xu M Y. On cubic s-arc transitive Cayley graphs of finite simple groups. Europ J Combin, 2005, 26: 133-143

[22] Xu S J, Fang X G, Wang J, Xu M Y. 5-arc transitive cubic Cayley graphs on finite simple groups. Europ J Combin, 2007, 28: 1023-1036

[23] Tutte W T. A family of cubical graphs. Proc Cambridge Philos Soc, 1947, 43: 459-474

[24] Tutte W T. On the symmetry of cubic graphs. Canad J Math, 1959, 11: 621-624 